\title{
Editorial
}

Pensar en Movimiento:

Revista de Ciencias del Ejercicio y la Salud EISSN 1659-4436

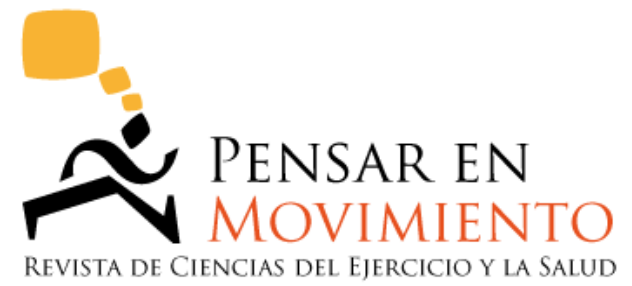

Vol. 17, No 2, pp. 1- 7

Inicia $1^{\circ}$ de julio, cierre al 31 de diciembre de 2019

\section{LA CIENCIA, Y LAS REVISTAS CIENTÍFICAS, SON UNA FARSA SI LA VERDAD NO EXISTE}

\author{
Luis Fernando Aragón-Vargas, Ph.D., FACSM \\ Centro de Investigación en Ciencias del Movimiento Humano \\ Universidad de Costa Rica, Costa Rica \\ Publicado: 2019-12-31 \\ DOI: https://doi.org/10.15517/pensarmov.v17i2.40253
}

He tenido el privilegio de compartir con varias docenas de colegas de la Universidad de Costa Rica, en los últimos dos años, un curso en línea para renovar la acreditación como investigadores con seres humanos. Uno de los módulos en este curso trata el tema de "El investigador como persona que busca la verdad". A lo largo de las interacciones con mis colegas, ha sido desconcertante para mí ver cómo muchos de ellos mantienen la posición de que no existe una verdad absoluta. ¿Por qué desconcertante? Por una razón muy sencilla: como lo propone el título de este editorial, si la verdad no existe, la ciencia y las revistas científicas son una farsa. A continuación, intentaré elaborar un poco esta idea, después de reflexionar brevemente sobre cómo llegamos al punto actual. Las reflexiones que voy a exponer se aplican de manera especial a las ciencias naturales, pero también son extrapolables a las ciencias sociales, con algunas consideraciones particulares.

"El punto actual", el ánimo de un sinnúmero de investigadores y académicos, se resume en una frase del Dr. Daniel Camacho: "El conocimiento es siempre provisional, no hay verdades absolutas" (Camacho, 2019). Ciertamente, a lo largo de la historia de las ciencias naturales, hemos aceptado como verdaderas muchas explicaciones que, aunque funcionaban bien, luego han resultado estar equivocadas. En una aparente muestra de humildad, hoy reconocemos que nuestras mejores explicaciones podrían dejar de ser válidas a mayor o menor plazo. Según el premio Nobel Sir Peter Medawar en su libro Los Límites de la Ciencia (Medawar, 1984), en la ciencia no puede haber certeza apodíctica, esto es, una certeza concluyente final fuera del alcance de la crítica. Por todo esto, no debería sorprendernos la afirmación del Dr. Camacho.

Así, una gran cantidad de investigadores estará de acuerdo con el comentario de mi colega -1 - 
Nelson Antonio Pérez Rojas en respuesta a mi pregunta ¿Buscamos los científicos la verdad, o nos interesa nada más encontrar explicaciones suficientemente buenas? Él responde: "Si se toma la 'verdad' como un consenso de los seres humanos que se encuentran en un entorno definido, o al menos como un acuerdo de la mayoría, en el presente podría considerarse 'verdad' algo que quizás no lo fue en el pasado ni lo será en el futuro (...) sabemos que, mientras 'la ciencia siga avanzando' o mientras la humanidad permanezca, se revelará información desconocida. En fin, buscamos verdades y revelar información que les permitirá a otros descubrir otras verdades en el futuro. Lo que parece imposible es creer en una verdad absoluta e inmutable."

La complejidad es mayor al considerar que el conocimiento de la verdad puede tener diferentes ópticas, pues quienes la buscamos somos, al fin y al cabo, seres humanos, permeados por nuestras realidades, experiencias, creencias, cultura, religión, etc. Un filósofo de la ciencia que exploró esto más en detalle fue Thomas Kuhn (2012; publicado originalmente en 1962), apuntando a una visión de la ciencia más subjetiva. Posteriormente, se ha visto la necesidad de una mayor objetividad, tomando en cuenta que el ser humano es un elemento ineludible, pero no indeseable, sino más bien esencial para la ciencia. "La filosofía de la ciencia contemporánea ha estado en la búsqueda de un término medio donde la razón, la observación y la objetividad tienen un lugar apropiado, pero donde el factor humano es al menos eso—un factor." (Ratzsch 2000, Dificultades con las perspectivas Kuhnianas).

Ahora bien: que no pueda haber certeza apodíctica en la ciencia (o en otros campos del conocimiento) no significa que la verdad no exista. El error en que podemos caer muy fácilmente es deducir que, en vista de que nuestro conocimiento de la verdad es imperfecto o incompleto, la verdad es siempre cambiante. Pero propongo ser más rigurosos en nuestro razonamiento: en cualquier argumento que se presente deberíamos aplicar, como mínimo, los principios de coherencia (consistencia interna) y correspondencia con la realidad. ¿No tiene acaso la afirmación "no hay verdades absolutas" un inconfundible tinte de verdad absoluta? Aquí falta coherencia. La posición tan generalizada de que "no hay verdades absolutas" no es coherente, se contradice a sí misma. Igual sucede cuando la gente dice que "no es posible llegar a la verdad", "todo es relativo" o "la verdad es siempre cambiante": se trata de afirmaciones que se contradicen a sí mismas. Cuando una frase se incluye en su campo de referencia, pero no se autosatisface, como en el caso de "no es posible llegar a la verdad", se refuta a sí misma. En ese caso, ¿podemos ser intelectualmente honestos si la aceptamos?

David Berlinski desarrolla esta idea un poquito, para ayudarnos con un asunto que puede ser difícil de digerir: "La tesis de que no existen las verdades absolutas-es acaso una verdad absoluta? Si lo es, entonces algunas verdades sí son absolutas después de todo y, si algunas lo son, ¿por qué no otras? Si no lo es, ¿por qué razón deberíamos prestarle atención, si la atención que nos merece va a variar según las circunstancias?" (Berlinski, 2009, p. 130).

En los últimos dos párrafos me he referido a la dificultad lógica de que no exista la verdad absoluta. Pero hay otras preguntas que es necesario plantearse. Por ejemplo: ¿hay acaso múltiples 
verdades, todas igualmente válidas? Hay quienes afirman que "la verdad no es única" y que, según los elementos utilizados, cada quien encontrará su propia verdad. Entonces, cuando dos o más "verdades" se contradicen, ¿tienen todas ellas la razón? Si todas las "verdades" (explicaciones) son igualmente aceptables, ¿tiene sentido investigar? Si más bien no todas las "verdades" tienen la razón, ¿cómo se decide quién la tiene? ¿La persona más locuaz? ¿La que tiene un laboratorio más caro? ¿La que logra más votos a favor en redes sociales? (cf. Aragón-Vargas, 2017). En el quehacer científico se definen criterios que nos ayudan a escoger entre las múltiples "verdades", con la idea de que la escogencia nos acerque a la verdad, en vez de que cada uno de nosotros tenga su propio argumento sofisticado, su propia "verdad" que no es ni puede ser mejor que la del vecino.

Se podría partir del supuesto de que cuanto más cerca está uno de la verdad, más exitoso es en sus predicciones al aplicar su teoría. Pero este último abordaje nos obligaría a aceptar que la verdad existe, es única y es inmutable, independientemente del conocimiento que tengamos de ella. Eso nos lleva a una perspectiva interesante, compartida por muchos investigadores y filósofos y de hecho fundamental en la historia de la ciencia, que nos presenta René Van Woudenberg (2017). Está resumida en dos presuposiciones metafísicas, es decir, acerca del mundo que, en sí, no pueden ser probadas científicamente: 1. El mundo exhibe un orden. 2. Las verdades acerca del mundo existen, independientemente de que sean conocidas o creídas por los seres humanos. Él plantea que sería incoherente ponerse a investigar el mundo físico y negar explícitamente que este es un mundo ordenado. Y yo añadiría: extremadamente complejo, ciertamente, pero ordenado y conocible. Si no es conocible, ¿para qué perder el tiempo investigando?

Hasta aquí, he propuesto que para el quehacer científico es necesario aceptar que existe una verdad, una realidad absoluta e inmutable que estamos intentado conocer. Al mismo tiempo, he reconocido que nuestro conocimiento de esa verdad, aunque es posible, no es ni puede ser perfecto o completo. Aquellas personas que me han acompañado hasta aquí se topan con una bifurcación en el camino, con dos guías distintos. Por una parte, podrían acompañar a Platón quien, en el Timeo o Timaeus, nos propone "...si podemos producir explicaciones no menos probables que cualquier otra, deberíamos darnos por satisfechos, recordando que tanto yo, el disertante, como ustedes, los jueces, somos solamente humanos (...) no nos corresponde buscar más allá de esto." (McGrew, AlspectorKelly \& Allhoff, 2009, p. 27). Ese sería el camino del científico que se contenta con una explicación suficientemente buena y que reconoce que habrá muchas otras igualmente buenas. O podrían optar por creer, conmigo, que la verdad existe y que, como científicos, es nuestro deber buscarla (con la colaboración de otras disciplinas). Aunque nunca podamos tener la certeza de haberla alcanzado, nosotros, y me atrevo a afirmar que una gran mayoría de investigadores, a menudo utilizamos terminología como "cuestionamientos más profundos", "progreso", "descubrimiento", "perfeccionamiento", "perfección del conocimiento" y "avance continuo", que sugieren el supuesto de que la verdad final sí existe y nos desplazamos hacia ella, aunque el acercamiento sea "como una asíntota" (Medawar, 1984). Sugieren que confiamos en que nuestros métodos son cada vez mejores; sugieren que el conocimiento que estamos construyendo es cada vez más completo, no simplemente cada vez más abundante. Como plantea Ratzsch: ““Quisiéramos creer que la ciencia

$-3-$

Esta obra está bajo una 
ha logrado progreso teórico a lo largo de los años y que ese progreso está ligado de alguna manera a la verdad. Nuestras teorías, en esta perspectiva, están más cerca de la verdad, o se aproximan más a la verdad, o tienen una mayor verosimilitud, que las teorías previas, aunque las teorías futuras podrían acercarse aún más." (Ratzsch, 2000, Realismo-Verdad Aproximada).

Existe una corriente en la filosofía de la ciencia que, a pesar de no tener todas las respuestas, es sólida en varios aspectos y tiene muchos elementos en común con lo que he planteado al final del párrafo anterior: el realismo científico. "Realismo es la perspectiva de que las teorías pueden ser descripciones verdaderas y acertadas de la realidad objetiva, que los términos téoricos pueden de hecho referirse a entidades reales que tienen (al menos algunas de) las propiedades que pensamos que tienen, que podemos conocer que algunas teorías son ciertas y que podemos conocer que las entidades y procesos a los que aspiran a referirse son efectivamente ciertos, y que tales descripciones y conocimiento son al menos a lo que apunta la ciencia." (Ratzsch, 2000, Realismo). El realismo tiene la limitación de que no podemos cotejar nuestras teorías con la realidad, para ver qué tanto se aproximan a ella, puesto que no tenemos acceso directo indiscutible a esa realidad. Sin embargo, tenemos una serie de recursos como la coherencia o consistencia, la simplicidad, la falseabilidad y la habilidad predictiva, que nos permiten tener mayor o menor grado de confianza en la correspondencia entre una determinada teoría y la realidad (Aragón-Vargas, 2017).

Dentro de las múltiples perspectivas filosóficas que se han ofrecido a lo largo de la historia de la ciencia, tantísimos ISMOS, yo quisiera ofrecer el yomismo científico como explicación inconsciente de la posición de muchos colegas investigadores, a saber, la imposibilidad de la existencia de una verdad absoluta. El yomismo propone que en el quehacer científico-sea de las ciencias naturales o las ciencias sociales-la fuerza principal, el impulso constante, es demostrar que yo tengo razón, o que el otro está equivocado. Lo paradójico es que al mismo tiempo que estoy convencido de tener la razón, me niego a reconocer que "mi razón" sea la verdad final o absoluta (nuevamente, una incoherencia). Cuando me sugieren que es posible encontrar la verdad, esa verdad se convierte en una amenaza, la cual debo evitar a toda costa pues corro el riesgo de no poder seguir investigando: quisiera mantener siempre una puerta abierta que me permita ofrecer una explicación distinta, novedosa. La búsqueda es apasionante, tanto, que pierdo la perspectiva del objetivo (el conocimiento) y centro todo el esfuerzo en el proceso. Cuando opera sutilmente en el subconsciente de los científicos, el yomismo nos traiciona y nos confunde. Pero no nos distraigamos con esta idea inédita, que podremos retomar en otro momento.

Vale la pena, en este punto del editorial, plantear algunas preguntas que nos lleven a analizar las implicaciones prácticas de una posición particular acerca de la verdad. Por ejemplo, si el universo es infinito y tiene un infinito número de situaciones, y "al ser infinito no podemos pensar en llegar a conocer y entender esa realidad", como me compartió una colega, ¿qué sentido tendría estudiar sus partes? Siempre alguien me podría contestar que la parte que estudié yo no es la importante, o la relevante, o la que está de moda. ¿No sería eso sumamente frustrante y fútil? Otro ejemplo: ¿sabemos los científicos que, como sostienen muchos, "ese universo físico al que llamamos realidad 
es cambiante"? Si es cambiante, no podemos hacer ciencia, pues una de las presuposiciones de la ciencia, como se planteó unos renglones atrás, es que el universo es un cosmos ordenado y regular. Pero no aceptemos la palabra de Van Woudenberg. Si el universo es cambiante, sólo podríamos buscar explicaciones suficientemente buenas... que además serían necesariamente temporales. ¿No sería esa una pérdida de tiempo? No tendríamos forma alguna de predecir la duración de su temporalidad, no podríamos medir la caducidad de cada explicación hasta que ya es demasiado tarde. ¡La ciencia perdería TODO su poder de predicción!

Podemos ir más allá en el análisis de estas consecuencias prácticas de las distintas posiciones filosóficas. Por ejemplo, algunos médicos argumentan que la verdad no existe pues, en su práctica clínica, los fenómenos no son siempre iguales, sin embargo, si la realidad que enfrenta un médico es totalmente distinta cada vez, entonces no puede encontrar patrones (ni vale la pena buscarlos). Eso significa que no pueden existir fundamentos para su práctica clínica, y el médico será un improvisador, con técnicas quizás sofisticadas y equipos caros a su disposición, pero improvisador al fin. Un mejor médico será alguien con más suerte, o "más artista", pues logra mejores resultados. En esta misma línea, un mejor científico no será más que alguien con más elocuencia para presentar su versión de la "verdad". En un mundo así, una vez obtenida la licencia para ejercer, no tendría sentido investigar ni seguir aprendiendo. Si más bien lo que sucede es que la realidad de una enfermedad o desorden sí existe, si el fenómeno es ordenado y consistente, aunque sea sumamente complejo y nuestro conocimiento al respecto sea incompleto o imperfecto, entonces sí vale la pena investigar, pues tendremos la esperanza de acercarnos cada vez más a la verdad.

Desde la perspectiva de las revistas científicas, este análisis adquiere una importancia enorme, pues debería haber coherencia entre la posición filosófica del ente editorial, y las políticas de ésta. Una revista podría sostener que el conocimiento debe ser pluralista, que todas las "verdades" son igualmente aceptables, que no hay una única forma correcta de hacer las cosas. Así, publicaría cualquier manuscrito que recibe, haciendo totalmente innecesaria la revisión por pares. En el mundo editorial estas revistas no son bien vistas; de hecho, muchas de ellas han sido calificadas despectivamente como "revistas depredadoras". En la realidad, las revistas que usted y yo respetamos definen criterios para decidir qué se debería publicar y qué no. Como se puede ver, no existe correspondencia entre la afirmación de que todas las "verdades" son igualmente aceptables y cómo funcionan las revistas científicas en la realidad.

Vayamos por un instante al extremo opuesto, a una revista que tenga una línea editorial muy definida, definida a tal extremo que es imposible publicar en ella cualquier hallazgo que se desvíe un nanómetro en cualquier dirección. La podríamos llamar obtusa, totalitaria, sesgada, subjetiva, rígida o tendenciosa, pero nunca científica. Su estilo estrujaría las herramientas que nos permiten aspirar a la objetividad, la racionalidad y el empiricismo, esos valores de la ciencia que tanto atesoramos.

Es aparente la necesidad de que las revistas científicas sean suficientemente rigurosas pero - 5 - 
abiertas. Como sostiene mi colega el Dr. Wálter Salazar, sea cual sea el estilo de comunicación de la investigación científica, siempre es necesario que otras personas (editores, revisores y la comunidad científica en general) puedan evaluar las ideas planteadas y la calidad del proceso. Nos apoyamos en herramientas que nos ayudan a discernir entre aproximaciones más acertadas y menos acertadas a la verdad. Nos adherimos a ciertas reglas o pautas que la comunidad científica ha escogido y que son de común aceptación. Las cuatro normas institucionales de la ciencia que nos propone Mariano Artigas, citando a Robert Merton en su artículo de 1942 "La estructura normativa de la ciencia", son solamente un ejemplo de estas pautas que deberían guiar la labor de las revistas científicas. Estas normas son: universalismo, que las afirmaciones de verdad deben ser independientes de la raza, nacionalidad, religión, clase, etc. (es una manera de decir que no hay múltiples verdades, todas ellas válidas, como he planteado en este editorial); comunismo, que se refiere a la propiedad comunal de los bienes, de modo que el conocimiento es propiedad común, no individual—el reconocimiento del científico depende de que comparta sus hallazgos, que los publique-(por esto es que publicamos los hallazgos y, además, por esto es tan importante el movimiento de acceso abierto a la información); el desinterés, que se refiere a patrones de control institucional, fundamentado en el carácter público y verificable de la ciencia (de nuevo, la publicación de los hallazgos, sujeta al escrutinio de pares o colegas en los distintos procesos de revisión, tanto antes como después de publicados); y al escepticismo organizado, que implica la suspensión temporal del juicio y el escrutinio desapegado de las creencias en términos de criterios empíricos y lógicos (se refiere a la obligación de editores y revisores de ser lo más objetivos posible) (Artigas, 2000, pp. 264-265).

En resumen, las normas y criterios que actualmente rigen a la ciencia y a las revistas científicas tienen sentido si consideramos que la verdad existe y la podemos conocer, que hay una mejor manera de hacer las cosas, que podemos completar cada vez más nuestro conocimiento y no simplemente aumentar la cantidad de información disponible. Si hay múltiples "verdades", todas igualmente válidas, la ciencia en general, y las revistas científicas actuales especialmente, no son más que un pasatiempo, un entretenimiento sumamente sofisticado y costoso, pero pasatiempo al fin. En ese caso, habría que admitir que son una farsa.

\section{Referencias}

Aragón-Vargas, L.F. (2017). ¿Qué es la ciencia y hasta dónde puede llegar? Pensar En Movimiento: Revista De Ciencias Del Ejercicio y La Salud, 15(1), 1-14. doi: https://doi.org/10.15517/pensarmov.v15i1.29637

Artigas, M. (2000). The Mind of the Universe. Radnor, PA: Templeton Foundation Press.

Berlinski, D. (2009). The Devil's Delusion. New York, NY: Random House, Inc.

Camacho, D. (2019). ¿Es la verdad sólo una ilusión?. Onda UNED, Costa Rica. Descargado el - 6 - 
23/12/19 de http://ondauned.com/transmision.php?ou=1388

Kuhn, T. S. (2012). The structure of scientific revolutions. Chicago, IL: The University of Chicago Press. Ed. $50^{\circ}$ Aniversario. [Versión Kindle para PC]. Recuperado de https://www.amazon.com/Structure-Scientific-Revolutions-50th-Anniversary-ebook-dpB007USH7J2/dp/B007USH7J2/ref=mt kindle? encoding=UTF8\&me=\&qid=1577739846

McGrew, T., Alspector-Kelly, M. y Allhoff, F. (2009). Philosophy of Science: an Historical Anthology. Oxford: Wiley-Blackwell.

Medawar, Peter B. (1984). The Limits of Science. New York: Harper and Row.

Ratzsch, D. (2000). Science \& its Limits: The Natural Sciences in Christian Perspective (2da ed.). Downers Grove, IL: InterVarsity Press. [Versión Kindle para PC]. Recuperado de https://www.amazon.com/Science-lts-Limits-Perspective-Philosophy-ebook/dp/B001Q3LA9K/

Van Woudenberg, R. (2017). Las presuposiciones de la ciencia (del movimiento humano). Pensar En Movimiento: Revista De Ciencias Del Ejercicio y La Salud, 15(1), 17-33. doi: https://doi.org/10.15517/pensarmov.v15i1.29046

Luis Fernando Aragón Vargas, Ph.D., FACSM

Director, PENSAR EN MOVIMIENTO

Universidad de Costa Rica 